\title{
Seasonal changes in physical qualities of elite youth soccer players according to maturity status: comparisons with aged matched controls
}

\author{
Rhys Morris ${ }^{a}$, Stacey Emmonds ${ }^{a}$, Ben Jones ${ }^{a}$, Tony D. Myers ${ }^{b}$, Neil D. Clarkec, Jason Laked, Matthew Ellise, \\ 5 Dave Singleton ${ }^{f}$, Gregory Roe ${ }^{\mathrm{a}}$ and Kevin Till ${ }^{\mathrm{a}}$
}

\begin{abstract}
anstitute for Sport and Physical Activity \& Leisure, Leeds Beckett University, Leeds, United Kingdom; ${ }^{b}$ Sport, Exercise and Health Research Centre, Newman University Birmingham, United Kingdom; 'School of Life Sciences, Coventry University, Coventry, United Kingdom; dDepartment, University of Chichester, Chichester, United Kingdom; ${ }^{e}$ Coventry City Football Club, Coventry, United Kingdom; 'Birmingham City Football Club, Birmingham, United Kingdom
\end{abstract}

\section{ABSTRACT}

Purpose:Longitudinal studies assessing the seasonal development of strength, speed and power qualities are limited in youth soccer players. The purpose of this study was to evaluate the seasonal changes in the physical development of elite youth soccer players across Pre-, Circa- and Post-Peak Height Velocity (PHV), against a similar age and maturity matched control groups. Methods:Onehundred and twelve male elite youth soccer players (Pre-PHV $n=55$; Circa-PHV $n=21$; Post-PHV $n=36$ ) and 38 controls consisting of non-elite active participants (Pre-PHV $n=18$; Circa-PHV $n=10$; Post-PHV $n=10$ ) all undertook isometric mid-thigh pull strength, 10-30 m sprints, change of direction speed (CODs) and countermovement jump (CMJ) tests pre- and post-season. Results:The elite CircaPHV improved greater than the control group for all physical qualities between pre- and post-season. The elite Pre-PHV improved greater in sprints, CODs, CMJ jump height and strength while the elite PostPHV group improved more in CODs and strength than their respective control groups. Conclusion: Findings suggest that systematic academy soccer training enhances the development of physical qualities in youth soccer players but maturity status may impact upon such adaptations.

\section{ARTICLE HISTORY}

Accepted 13 March 2018

\section{KEYWORDS}

Strength; youth; seasonal;

changes; maturation; soccer

\section{Introduction}

Soccer is an intermittent team sport placing physiological demands on both the aerobic and anaerobic energy systems (Morgans et al. 2014). It has been suggested that the anaerobic system, including physical qualities such as strength, speed and power, are instrumental for both general movement patterns (e.g., jumping) and within-match outcomes (e.g., making a tackle) in soccer (Deprez et al. 2013). It is therefore common practice to monitor and evaluate the development of these physical qualities (Williams et al. 2011) with a range of research presenting the physical qualities of youth soccer players using cross-sectional methodologies (Lovell et al. 2015; Emmonds et al. 2016; Towlson et al. 2017). Cross sectional methods allow researchers and practitioners to assess and evaluate at a specific time point but fail to provide any insight into long-term development and change in performance (Cobley and Till 2017). From a research standpoint, the necessity of longitudinal design can allow practitioners to identify causal effect, developmental changes and interactions between variables within youth athletes (Valente-dosSantos et al. 2012; Till et al. 2013; Cobley and Till 2017).

Longitudinal analysis allows practitioners to gain an understanding of the physical development and specifically, understand the impact a training environment may have on the development of physical qualities (Cobley and Till 2017). To date there are limited studies available tracking the development of physical qualities within youth soccer players over a competitive season. Recent longitudinal studies in youth soccer players (Vänttinen et al. 2011; Hammami et al. 2013) have identified improvements in a range of parameters (e.g., $30 \mathrm{~m}$ sprint, jump performance). Both studies concluded that the elite youth soccer players were physically superior to their respective control groups, attributing this to the direct consequence of the soccer training environment the elite players were exposed to. To build on these findings, controlling for baseline performance and maturation status within statistical models, may shed some insight into the impact a training environment may have irrespective of natural growth and maturation (Lloyd et al. 2014a; Meyers et al. 2017).

Recently, Wrigley et al. (2014) identified the impact training and maturation had on the development of physical qualities (i.e., speed, power and aerobic fitness) within elite youth soccer players, by statistically controlling for baseline performance and changes in maturation over a 3-year period. Findings demonstrated accelerated physical development over a 3-year period within English academy soccer players. Future research should try and differentiate between the maturation status of participants (i.e., Pre-Peak Height Velocity [PHV], Circa-PHV, Post-PHV), to truly understand the impact upon physical development changes throughout childhood and adolescence (Ford et al. 2011). Furthermore, a more holistic testing battery should be considered, that includes qualities that are important for soccer success (i.e., strength) 


\section{Participants}

A total of 112 male elite youth soccer players aged 12-18 years (Pre-PHV $n=55$; Circa-PHV $n=21$; Post-PHV $n=36$; see Table 1) were recruited from 4 professional soccer academies. A control group consisting of non-elite active participants (Pre-PHV $n=18$; Circa-PHV $n=10$; Post-PHV $n=10$; see Table 1) also participated in the study. The elite group were involved in a full time professional soccer academy. The Pre-PHV and Circa-PHV participants undertook on average 4

110 football training sessions, and 1-2 strength and conditioning sessions per week. The Post-PHV group were involved in 2-3 strength and conditioning sessions on average, per week with 6 football sessions. Each maturity group also competed in 1 soccer match per week throughout the season. The control group were school children who participated in 2 schoolbased sessions of physical activity per week ( $2 \mathrm{~h}$ in total) and did not take part in any extracurricular sport outside of school. All experimental procedures gained institutional ethics approval with informed and parental written consent obtained.

\section{Procedures}

Anthropometric and physical qualities were collected at the start (i.e., September) and end (i.e., May) of the 2015-2016 soccer season. All testing took place on an artificial $3 \mathrm{G}$ playing surface in an indoor facility and was conducted at least $48 \mathrm{~h}$ post competitive match-play or strenuous training for all participants. Prior to testing, participants performed a standardized $10 \mathrm{~min}$ warm-up consisting of jogging, dynamic stretching and acceleration drills. The elite group were familiar with the testing battery except the isometric mid-thigh pull (IMTP) strength assessment. All participants undertook a familiarization testing session before the study commenced including 3 attempts of the testing battery (all measures).

\section{Anthropometry}

Height and sitting height were measured to the nearest $0.1 \mathrm{~cm}$ using a Seca Alpha stadiometer(Seca, Hamburg, Germany). Body mass was determined from body weight and taken to be BW $\cdot g^{-1}(\mathrm{~kg})$ with $g=$ acceleration due to gravity measured on a commercially available portable force platform (AccuPower, AMTI, ACP, Watertown, MA, USA) using a sampling rate of $400 \mathrm{~Hz}$ then multiplied by 9.81 to convert to $\mathrm{kg}$.

\section{Maturity offset}

Age at PHV was estimated by the Mirwald prediction equation (Mirwald et al. 2002). Years from PHV (YPHV) were calculated for each participant by subtracting the age at PHV from chronological age with a \pm 6 month error rate. Participants were allocated to either Pre-PHV (offset $<-1$ years), CircaPHV (between -1 to + years) or Post-PHV ( $>+1$ years) groups in relation to their YPHV at the pre-testing session based on previous research (Wrigley et al. 2014). Changes in maturation were determined by calculating the difference between the start and end of season change in relation to YPHV (Wrigley et al. 2014).

\section{Isometric mid-thigh pull (IMTP)}

The IMTP was utilised as a measure of lower body strength. The IMTP was performed on a commercially available portable force platform (AccuPower, AMTI, ACP, Watertown, MA, USA) and recorded vertical force at $1000 \mathrm{~Hz}$. Participants performed the IMTP on a customized pull rack with their shoulders placed over the bar in a position similar to that of the second pull of a power clean (Haff et al. 2015). Participants performed 2 IMTP trials, each lasting $6 \mathrm{~s}$, with $5 \mathrm{~min}$ ' rest between trials. The IMTP start was identified using a 5 SD threshold that was calculated from $1 \mathrm{~s}$ of quiet standing force recorded before the start of each pull (Dos'Santos et al. 2016). Participants were instructed to pull as "fast and hard" as possible, and received loud verbal encouragement (Dos'Santos et al. 2016). Each participant's best trial, as determined by the highest peak force (PF), was selected for analysis. Relative peak force (rPF) was calculated as PF/body mass. Intraclass correlation coefficient (ICC) and coefficient of variation (CV) for the IMTP PF and rPF were $r=0.98$ and $\mathrm{CV}=4.91 \%$.

\section{Countermovement jump (CMJ)}

The CMJ utilized as a measurement for lower body power. The CMJ was performed on the same portable force platform sampling vertical force $(\mathrm{Fz})$ at $1000 \mathrm{~Hz}$. After a $1 \mathrm{~s}$ quiet standing period, CMJ was performed utilising a standard technique with arms akimbo (Hori et al. 2007), with no attempts made to control the depth of the countermovement (Mundy et al. 2017). Each participant performed 2 jumps interspersed 
with 3 min rest. Jump height was calculated using the velocity at take-off method (Mundy et al. 2017) whilst net force was integrated with respect to time to obtain net impulse which was summed over the propulsion phase. ICCs and CVs for the CMJ jump height were $r=0.86$ and $\mathrm{CV}=7.46 \%$ and $\mathrm{CMJ}$ impulse were $r=0.94$ and $\mathrm{CV}=6.67 \%$.

\section{Sprint performance}

For sprint performance, distances of 10 and $30 \mathrm{~m}$ were assessed using Brower photocell timing gates (model number

190 BRO001; Brower, Draper, UT, USA). All participants performed 2 trials, with 3-5 min of rest between trials. Athletes started 0.5 $\mathrm{m}$ behind the first gate from a 2-point staggered start (Thomas et al. 2015). The best performance from each of the 2 trials was used for analysis. ICCs and CVs for $10 \mathrm{~m}$ were $r=0.84$ and $\mathrm{CV}=3.61 \%$ and $30 \mathrm{~m}$ were $r=0.81$ and $\mathrm{CV}=2.53 \%$.

\section{Change of direction speed (CODs)}

The Arrow Head Agility Test (Noon et al. 2015) was used to assess COD using Brower photocell timing gates with 2 trials performed each side with 3-5 min rest between trials. The fastest time achieved from both sides was used for analysis.

ICCs and CVs for left side were $r=0.93$ and CV $=2.53 \%$ and right side were $r=0.92$ and $\mathrm{CV}=2.16 \%$.

\section{Statistical analyses}

Differences between the elite and control group's end of season measures were analysed using an Objective Bayesian regression model, with group (elite and control) as a categorical predictor, and both baseline score and the changes in maturation included as covariates. This analysis can be interpreted in the same way as a traditional analysis of covariance, but the $P$-values presented are the probability of the difference between groups, larger $P$-values relating to a higher probability of a difference. Estimated marginal means displayed represent the predicted differences between groups adjusted for the covariates. To illustrate the uncertainty around the estimation, lower and upper 95\% Higher Density Intervals (HDI) are reported. HDIs somewhat equate to traditional confidence intervals, but are more intuitively interpreted, as a $95 \%$ chance that a true difference is found in the HDI. They also provide distributional information rather than a mere interval of equally probable values (as is the case with traditional confidence intervals).

Population effect sizes (delta) rather than sample effects (Cohen's D) were calculated using MCMC (Markov Chain Monte (arlo) estimation and illustrated the uncertainty of this estimate with lower and upper 95\% HDls. Effect size (ES) values of $0.2,0.5$ and 0.8 were considered to represent small, moderate and large differences respectively (Cohen 1988). A Bayesian formulation for quantifying and interpreting the magnitude of effect and "smallest worthwhile change" (SWC) was employed (Mengersen et al. 2016). The SWC used was a standardised change of 0.2 based on previous recommendations (Hopkins et al. 2009). All analyses were conducted using R (R Core Team 2016; using MCMCpack (Martin et al. 2011).

\section{Results}

The mean and SD's for anthropometric and maturation characteristics (Table 1) and physical qualities (Table 2) are presented pre and post season for the elite and control groups according to maturity group. Tables 3-5 report the Bayesian regression model analysis for the seasonal development of physical qualities between elite and control groups according to maturity status.

\section{Pre-PHV}

The Pre-PHV elite group improved more than the control group in all physical qualities except for CMJ impulse. Small ES were seen for $10 \mathrm{~m}(\mathrm{ES}=-0.31)$, and $30 \mathrm{~m}(\mathrm{ES}=-0.09)$ sprint performance, jump height $(E S=-0.19)$ and $P F(E S=0.14)$. A moderate ES was seen for $\mathrm{rPF}(\mathrm{ES}=0.58)$. Large ES were observed for CODs left $(E S=-1.07)$ and CODs right $(E S=)$. The probabilities of achieving greater than the SWC for CMJ impulse and jump height are highly uncertain whilst 10-30 m sprint performance, CODs and strength are highly probable (Table 3 ).

\section{Circa-PHV}

The Circa-PHV elite group improved more than the control group for all physical qualities.

Small ES were seen for jump height $(E S=0.34)$, CMJ Impulse (ES = 0.09) and PF (ES = 0.17).

Moderate ES are demonstrated for $10 \mathrm{~m}$ (ES $=-0.77$ ), $30 \mathrm{~m}$ $(E S=-0.58)$ sprint performance, and $\mathrm{rPF}(E S=0.59)$. Large $E S$ were seen for CODs left ( $E S=-1.31)$ and CODs right $(E S=-1.32)$. The probabilities of achieving changes greater than the SWC are uncertain for $C M J$ Impulse $(P=0.39)$ and PF $(P=0.45)$ whilst the rest of the variables are highly probable (Table 4$)$.

\section{Post-PHV}

For Post-PHV, the control group improved greater, all be it with a trivial $E S$ in $C M J$ impulse $(E S=-0.06)$ and small ES in 10 $m(E S=-0.22)$ and $30 m(E S=0.30)$ sprint performance. The elite Post-PHV group improved greater in PF $(E S=0.19)$ and rPF (ES = 0.03) but with trivial ES. The elite group also improved greatest in CODs left (ES $=-0.47)$ and right $(E S=-0.42)$ with small ES. The probabilities of achieving changes greater than the SWC for the elite group are uncertain for sprint performance, CMJ and rPF (Table 5). There are very highly probable changes, greater than the SWC, demonstrated by the elite group in CODs and PF. Jump height did not improve greater by either the control or the elite group.

\section{Discussion}

The purpose of this study was to evaluate the seasonal changes in the physical qualities of elite youth soccer across Pre-, Circa- and Post-PHV maturity groups against a control group, whilst controlling for baseline performance and changes in maturation status. The elite Pre-PHV group improved by a greater margin in 10-30 m sprint performance, CODs, jump height, PF and rPF than the control group. The 


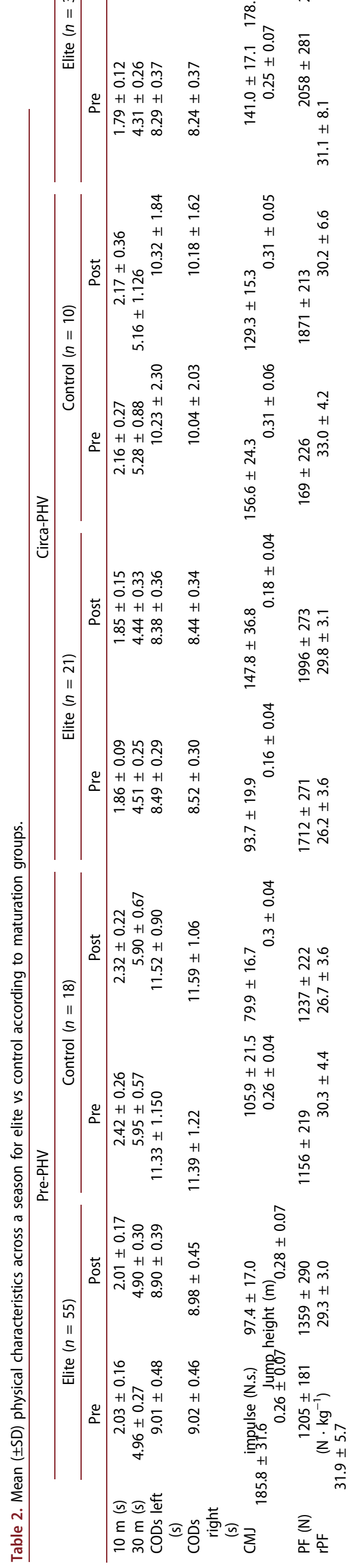

SCIENCE AND MEDICINE IN FOOTBALL 5 


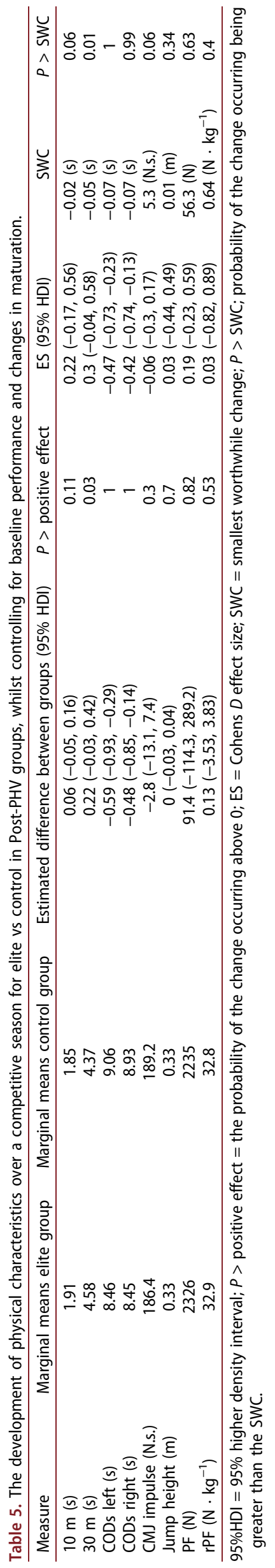

elite Circa-PHV improved across all variables whilst the postPHV group only improved in CODs, PF and rPF. The inclusion of the control group may represent a general population distribution (Page 2012) which could allow us to speculate that the training exposure has had an impact on strength, speed and power development for Pre- and Circa-PHV groups, but these may be reduced during Post-PHV.

\section{Pre-PHV}

The elite Pre-PHV group improved greater in 10 and $30 \mathrm{~m}$ sprint performance than the control group. These changes are highly probable to occur over the course of the season and greater than the SWC thresholds. These findings are consistent with previous research in U12 (Vänttinen et al. 2011), U14 (Hammami et al. 2013) and U12-U14 (Huijgen et al. 2010) in youth soccer players. However, specific mechanisms other than training exposure were not discussed and these studies either failed to account for maturation, consider maturity groups (Pre-, Circa-, Post-PHV), or in some cases, failed to provide a control group (Huijgen et al. 2010), so caution is needed when making comparisons. However, speed development happens in a non-linear fashion throughout adolescence and maturation plays a key role (Meyers et al. 2015). It has also been suggested that the ground contact phase should be of priority to elicit improvements in step frequency and ultimately improve speed, as changes will not occur naturally during growth and maturation in this phase of running (Meyers et al. 2016). Therefore, it is plausible the exposure to soccer match play and training with the added strength and conditioning sessions (1-2 per week) in the elite group, elicit these enhancements in ground contact, increasing step frequency and ultimately improving sprint performance when compared to the control group.

When we consider lower-body power, to the authors knowledge this is the first study to report the development of jumping performance using force-time characteristic over a competitive season in Pre-PHV athletes so direct comparisons are difficult. Firstly, when maturation is not controlled for, the development in jump height is greatest by the Pre-PHV elite group compared to the control group. These findings support those reported by Philippaerts et al. (2006) and Lloyd et al. (2011). However, when we control for changes in maturation, these data demonstrate superior improvements only in jump height for the elite group and not for concentric impulse. The probability of the elite group improving greater than the control group in jump height is in fact questionable when we consider the SWC and any difference above 0 $(P>$ effect). Therefore, unlike speed development, the soccer training did not elicit improvements in all CMJ variables and it is likely jump height changes are a consequence of growth and maturation as demonstrated by the mean differences when maturation is considered.

The development of strength for the Pre-PHV elite group outperformed that of the control group with high probabilities of the change occurring (see Table 3). To the authors' knowledge, this is the first study to report IMTP strength development in Pre-PHV male athletes. The recent shift in the paradigm of youth physical development advocates early 
participation in youth strength and conditioning with benefits being reported as early as 7 years of age (Faigenbaum et al. 2013). These benefits can be associated with neural changes, such as increased synchronization and firing rates, owing to the lack of androgen hormones in this stage of development (Lloyd et al. 2014b). Therefore, similar to the development of speed, the academy soccer training improved strength irrespective of changes in maturation. These mechanisms can potentially be attributed to the myelination and enhancement of the neural muscular system as a result of the training exposure and potential increases in muscle mass, although this mechanism may be unlikely (Lloyd et al. 2015).

\section{Circa-PHV}

The elite Circa-PHV group outperformed the control group for changes in all physical qualities. During the point leading up to and after PHV, concentrations in hormones, such as, growth hormone, insulin growth factor 1 and testosterone increase, which mediate the anabolic effect following physical activity (Rogol et al. 2002). Although hormone concentrations were not measured, the physical improvements demonstrated by the elite Circa-PHV group can be attributed to training induced adaptations that are then accelerated by the increased hormone concentrations (Matos and Winsley 2007). These observations may serve to highlight the importance of an early introduction into resistance training for boys wishing to enhance their maximal speed, CMJ performance and strength based on the current elite group undertaking strength and conditioning training (1-2 sessions per week). For example, research has demonstrated speed development is greatest during the maturation growth spurt (PHV) (Hirose 2009; Huijgen et al. 2010; Hirose and Seki 2016; Meyers et al. 2017) and in some instances the Circa-PHV (growth spurt period) improved twice as much as the Post-PHV group (Hirose and Seki2106) ) and those who remain in Pre-PHV (Lloyd et al. 2016). Therefore, Circa-PHV may be a sensitive period of time and an opportunity to exploit to improve speed development. Finally, Wrigley et al. (2014) also demonstrated enhanced speed development for academy players vs. nonacademy players with a weekly training load ( 2000 AU and $\sim 300 \mathrm{AU}$ ) and suggested the advancements seen by the academy players were a consequence of training.

To our knowledge this is the first study to provide forcetime data (net impulse and jump height) over a competitive season in Circa-PHV elite youth soccer players compared to a control group. The only elite group to demonstrate superior improvement in CMJ performance was the Circa-PHV group. However, as discussed earlier, there are suggestions of a sensitive period for CMJ development, similar to speed development (Philippaerts et al. 2006). For example, Malina et al. (2004) suggests a period around PHV also accelerates the development of vertical power, which is apparent within the current study's findings. Wrigley et al. (2014) then reports very similar findings with superior development in the elite academy players as a collective maturation albeit with a moderate effect. Although direct mechanisms were not discussed in the above studies and lacking in this study, it is possible the elite Circa-PHV group reap the benefits associated with the interaction between circulating androgen hormones and training, further research should look to address these potential mechanisms. Nonetheless, Wrigley et al. (2014) explicitly states the superior development of the academy soccer players is a direct consequence of the systematic training.

The development of IMTP strength in Circa-PHV demonstrates superior improvement by the elite groups. Vänttinen et al. (2011) investigated the longitudinal development of isometric maximal strength (leg extensors, abdominal, back, grip) in Norwegian soccer players compared to a control group, across 3 age groups (10.8 $\pm 0.3 ; 12.7 \pm 0.2 ; 14.7 \pm 0.3)$ over 2-year period. They reported the older age groups, that were exposed to strength training, had significantly greater strength levels than their age matched control group. However, the younger soccer players, that were not exposed to resistance training, did not differ compared to the control. Vänttinen et al. (2011) states these findings were a consequence of the specificity of training (resistance) and the natural rise in sex hormones associated with anabolic effects on performance, experienced by the soccer group. This information supports the beneficial effect training exposure has on the strength development in youth soccer players compared to a control group around the period of PHV.

\section{Post-PHV}

In contrast to the Pre- and Circa-PHV groups, the Post-PHV control group improved 10 and $30 \mathrm{~m}$ sprint performance more than the elite group over the season. Two potential explanations for these findings include: (1) inadequate training stimulus and (2) the accumulation of fatigue within a professional soccer academy. The current training and playing schedule of the U18's (Post-PHV) group consists of full time training 5 days per week; 1 competitive match. Similarly, a marked rise in training exposure and accumulation of fatigue has been shown to limit the development of speed post 16 years of age (akin to Post-PHV) (Huijgen et al. 2010). As a result, Gabbett et al. (2014) warns against high workloads in the developing athlete, irrespective of their responsiveness of training during maturation, as this may be detrimental to development. This detriment has been shown to effect specific anaerobic qualities such as sprint speed, CODs and CMJ performance (Noon et al. 2015) but not aerobic capacities, such as YOYO scores, in youth soccer players (Clark et al. 2008; Huijgen et al. 2010; Noon et al. 2015). Noon et al. (2015) reported decreases in sprint, arrow head and CMJ performance as the season progressed in elite youth soccer players (age $=17 \pm 1$ years) with an average training exposure of $9.6 \mathrm{~h}( \pm 2.9 \mathrm{~h})$. Although training load was not accounted for in the current study, it could be plausible that the neuromuscular and strength training stimuli the Post-PHV group were exposed to may not have been sufficient to stimulate adaptations (Noon et al. 2015) with the combining factors of the high intensity, aerobic nature of soccer training, resulting in a shift in endurance capabilities and diminishing the explosive capabilities of the soccer players within the study (Noon et al. 2015). This would explain why superior development in sprint speed and jump height was demonstrated by the control group over the course of the season. 
Finally, strength performance over the competitive season was improved greatest by the elite Post-PHV group, however with questionable probability values regarding rPF. When we consider the SWC threshold for rPF, the change over the course of the season is uncertain. These finding are in line with those reported by (Vänttinen et al. 2011) who suggested the specificity of resistance training and rise in hormones enhanced the development of strength. Unlike the explosive qualities that diminished for the elite Post-PHV group discussed earlier (sprint speed, CMJ, CODs), the IMTP PF is a measure of maximum strength and it is plausible this may not be impeded by the high workloads and shift in aerobic capacities during the course of the season. This idea can be supported by the findings from Häkkinen et al. (2003) who reported significant increases in maximum strength for both, strength and endurance group and strength group alone, during

470 a 21-week training intervention. Although both groups demonstrated increases in maximum strength, only the strength group improved in force at $500 \mathrm{~ms}$ and rate of force development at $500 \mathrm{~ms}$. These time sensitive variables are associated with neuromuscular function, similar to that of the CMJ and sprint performance and are seen as explosive qualities. Häkkinen et al. (2003) suggested the explosive, time sensitive qualities, were affected as a consequence of the endurance training. It is possible this concept may have contributed to the decrements in $\mathrm{CMJ}$ and sprint performance and not in maximum strength (PF, rPF), over the 480 course of the season for the elite Post-PHV group. Although this is plausible it must be highlighted that the participants used by Häkkinen et al. (2003) were described as recreational participants of physical activity, so they may be more sensitive and respondent to training stimuli than the current elite group.

\section{Limitations}

Although this study enhances on previous studies evaluating seasonal changes in youth soccer players according to maturity status it is not without limitations. Firstly, training load data was not obtained, which may have offered some explanation for the physical changes that occurred. Secondly, the use of the Mirwald equation to assess maturity status has previously been questioned due to its potential associated error of \pm 6 months. Next, the sample size of the control group was not equal to that of the elite group and may not have truly represented an aged and maturity matched population (e.g., the elite Pre-PHV group were older than the control group) but such recruitment is difficult within controlled based studies. Finally, although the study design was over a season, increasing the number of testing sessions would also serve to provide more information on the potential development and regression in development over the course of the season. This multi-longitudinal approach would serve to answer more questions (i.e., accumulation of fatigue vs inadequate stimuli). Multiple testing points may also identify the impact sensitive periods such as pre-season and its impact on performance where training load and volume is substantially increased.

\section{Conclusion}

By comparing changes in the physical qualities of an elite and control group over a season we found, when adjusting for baseline performance and changes in maturity status, the degree to which a season of elite academy training enhances the rate of change in performance across a range of indicators of physical qualities in elite players. These improvements are not associated with natural growth, maturation and development. Academy soccer training had a consistent effect on PrePHV and Circa-PHV groups when we consider 10-30 m sprint performance, CODs and some jump heights, all showing improvements. However, less consistent findings were shown for the elite Post-PHV group as they failed to improve in sprint speed and CMJ impulse.

Practical Implications

Such findings suggest that academy training for Pre- and Circa-PHV will enhance physical performance compared to school age and maturity matched individuals but more careful consideration for the physical development of the Post-PHV soccer players may be required given the large training volumes undertaken within soccer academies at this age or the lack of specificity within their training programs. These findings have implications for soccer coaches, sport scientists and strength and conditioning coaches in maximising the physical development of youth soccer players for successful long-term athlete development.

\section{Disclosure statement}

No potential conflict of interest was reported by the authors.

\section{References}

Clark NA, Edwards AM, Morton RH, Butterly RJ. 2008. Season-to-season variations of physiological fitness within a squad of professional male soccer players. J Sports Sci Med. 7(1):157.

Cobley S, Till K. 2017. Longitudinal tracking of athlete development: its importance, methods \& future considerations. Chapter 18. In: Baker, J, Cobley, S, Schorer, J, Wattie, N, editors. The handbook of talent identification and development in sport. London. Routledge; p. 248-266.

Cohen J. 1988. Statistical power analysis for the behavioral sciences. 2nd ed. Hillsdale: Lawrence Erlbaum.

Deprez D, Coutts AJ, Frnasen J, Deconick M, Lenoir M, Vayens R, Philippaerts R. 2013. Relative age, biological maturation and anaerobic characteristics in elite youth soccer players. Int J Sports Med. 34:897-903.

Dos'Santos T, Jones PA, Comfort P, Thomas C. 2017. Effect of Different Onset Thresholds on Isometric Midthigh Pull Force-Time Variables. The Journal of Strength \& Conditioning Research, 31(12), 3463-3473. .

Emmonds S, Till K, Jones B, Mellis M, Pears M. 2016. Anthropometric, speed and endurance characteristics of English academy soccer players: do they influence obtaining a professional contract at 18 years of age? Int J Sports Sci Coach. 11(2):212-218.

Faigenbaum AD, Farrell AC, Fabiano M, Radler TA, Naclerio F, Ratamess NA, Myer GD. 2013. Effects of detraining on fitness performance in 7 year-old children. J Strength Cond Res. 27(2):323-330.

Gabbett TJ, Whyte DG, Hartwig TB, Wescombe H, Naughton GA. 2014. The relationship between workloads, physical performance, injury and illness in adolescent male football players. Sports Med. 44(7):989-1003.

Haff GG, Ruben RP, Lider J, Twine C, Cormie P. 2015. A comparison of methods for determining the rate of force development during isometric midthigh clean pulls. J Strength Cond Res. 29(2):386-395.

Häkkinen K, Alen M, Kraemer WJ, Gorostiaga E, Izquierdo M, Rusko $H$, Mikkola J, Häkkinen A, Valkeinen H, Kaarakainen E, et al. 2003. 
Neuromuscular adaptations during concurrent strength and endurance training versus strength training. Eur J Appl Physiol. 89(1):42-52.

Hammami MA, Abderrahmane A, Nebigh A, Le Moal E, Ounis BO, Tabka Z, Zouhal H. 2013. Effects of a soccer season on anthropometric characteristics and physical fitness in elite young soccer players. J Sports Sci. 31(6):589-596.

Hirose N. 2009. Relationships among birth-month distribution, skeletal age and anthropometric characteristics in adolescent elite soccer players. J Sports Sci. 27(11):1159-1166.

Hirose N, Seki T. 2016. Two-year changes in anthropometric and motor ability values as talent identification indexes in youth soccer players. $J$ Sci Med Sport. 19(2):158-162.

Hopkins WG, Marshall SW, Batterham AM, Hanin J. 2009. Progressive statistics for studies in sports medicine and exercise science. Med Sci Sports Exerc. 41(1):3-13.

Hori N, Newton RU, Andrews WA, Kawamori N. 2007. Comparison of four different methods to measure power output during the hang power clean and the weighted jump squat. J Strength Cond Res. 21 (2):314.

Huijgen BC, Elferink-Gemser MT, Post W, Visscher C. 2010. Development of dribbling in talented youth soccer players aged 12-19 years: a longitudinal study. J Sports Sci. 28(7):689-698.

Lloyd RS, Faigenbaum AD, Stone MH, Oliver JL, Jeffreys I, Moody JA, Brewer C, Pierce KC, McCambridge TM, Howard R, et al. 2014a. Position statement on youth resistance training: the 2014 International Consensus. Br J Sports Med. 48(7):498-505.

Lloyd RS, Meyers RW, Hughes MG, Cronin JB, Oliver JL. 2016. The relationship between vertical and horizontal stiffness and maximal running speed in 11-15 year old boys. J Strength Cond Res. 30:S99.

Lloyd RS, Oliver JL, Faigenbaum AD, Myer GD, Croix MBDS. 2014b. Chronological age vs. biological maturation: implications for exercise programming in youth. J Strength Cond Res. 28(5):1454-1464.

Lovell R, Towlson C, Parkin G, Portas M, Vaeyens R, Cobley S. 2015. Soccer player characteristics in English lower-league development programmes: the relationships between relative age, maturation, anthropometry and physical fitness. PLoS ONE. 10(9):e0137238.

Malina RM, Eisenmann JC, Cumming SP, Ribeir B, Aroso J. 2004. Maturityassociated variation in the growth and functional capacities of youth football (soccer) players 13-15 years. Eur J Appl Physiol. 91(5-6):555-562.

Martin A, Quinn KM, Park JH. 2011. MCMCpack: Markov Chain Monte Carlo in R. J Stat Softw. 42(9):1-21. http://www.jstatsoft.org/v42/i09/

Matos N, Winsley RJ. 2007. Trainability of young athletes and overtraining. J Sports Sci Med. 6(3):353.

Mengersen KL, Drovandi CC, Robert CP, Pyne DB, Gore CJ. 2016. Bayesian estimation of small effects in exercise and sports science. PLoS ONE. 11 (4):e0147311.

Meyers RW, Oliver JL, Hughes MG, Lloyd RS, Cronin JB. 2017. Influence of age, maturity, and body size on the spatiotemporal determinants of maximal sprint speed in boys. J Strength Cond Res. 31(4):1009-1016.
Mirwald RL, Baxter-Jones AD, Bailey DA, Beunen GP. 2002. An assessment of maturity from anthropometric measurements. Med Sci Sports Exerc. 34(4):689-694.

Morgans R, Orme P, Anderson L, Drust B. 2014. Principles and practices of training for soccer. J Sport Health Sci. 3(4):251-257.

Mundy PD, Smith NA, Lauder MA, Lake JP. 2017. The effects of barbell load on countermovement vertical jump power and net impulse. J Sports Sci. 35(18):1781-1787.

Noon MR, James RS, Clarke ND, Akubat I, Thake CD. 2015. Perceptions of well-being and physical performance in English elite youth footballers across a season. J Sports Sci. 33(20):2106-2115.

Page P. 2012. Research designs in physical therapy. Int J Sport Phys Ther. 7 (5):482-492.

Philippaerts RM, Vaeyens R, Janssens M, Van Renterghem B, Matthys D, Craen R, Malina RM. 2006. The relationship between peak height velocity and physical performance in youth soccer players. J Sports Sci. 24 (3):221-230.

R Core Team. 2016. R: a language and environment for statistical computing. Vienna (Austria): R Foundation for Statistical Computing. https:// www.R-project.org/

Rogol AD, Roemmich JN, Clark PA. 2002. Growth at puberty. J Adolesc Health. 31(6):192-200.

Thomas C, Comfort P, Chiang CY, Jones PA. 2015. Relationship between isometric mid-thigh pull variables and sprint and change of direction performance in collegiate athletes. J Trainol. 4(1):6-10.

Till K, Cobley S, O'Hara J, Chapman C, Cooke C. 2013. A longitudinal evaluation of anthropometric and fitness characteristics in junior rugby league players considering playing position and selection level. J Sci Med Sport. 16(5):438-443.

Towlson C, Cobley S, Midgley AW, Garrett A, Parkin G, Lovell R. 2017. Relative age, maturation and physical biases on position allocation in elite-youth soccer. Int J Sports Med. 38(03):201-209.

Valente-dos-Santos J, Coelho-e-Silva M, Severino V, Duarte J, Martins RS, Figueiredo AJ, Malina RM. 2012. Longitudinal study of repeated sprint performance in youth soccer players of contrasting skeletal maturity status. J Sports Sci Med. 11(3):371-379.

Vänttinen T, Blomqvist M, Nyman K, Häkkinen K. 2011. Changes in body composition, hormonal status, and physical fitness in 11-, 13-, and 15year-old Finnish regional youth soccer players during a two-year follow-up. J Strength Cond Res. 25(12):3342-3351.

Williams CA, Oliver JL, Faulkner J. 2011. Seasonal monitoring of sprint and jump performance in a soccer youth academy. Int J Sports Physiol Perform. 6(2):264-275.

Wrigley RD, Drust B, Stratton G, Atkinson G, Gregson W. 2014. Long-term soccer-specific training enhances the rate of physical development of academy soccer players independent of maturation status. Int J Sports Med. 35(13):1090-1094. 